\title{
Desigualdades sociales y tipos de territorios en Chile*
}

\author{
Óscar Mac-Clure
}

Universidad de Los Lagos, Santiago, Chile. Email: oscar.macclure@ulagos.cl

\section{Rubén Calvo}

Universidad de Chile, Santiago, Chile. Email: rcalvo.gallardo@gmail.com

Resumen: Las desigualdades sociales en Chile incluyen desigualdades territoriales que permanecen no observadas cuando se enfoca exclusivamente las regiones administrativas. Un conjunto entrelazado de tipos de territorios representativos del proceso de urbanización en un nivel subregional, es propuesto para el estudio de las desigualdades sociales desde una perspectiva territorial. Un territorio es delimitado por un mercado de trabajo común, lo que es medido por la conmutación o movilidad territorial.

Palabras claves: desigualdad, urbanización , mercado de trabajo, territorios, conmutación.

\section{Social inequalities and types of territories in Chile}

Abstract: Social inequalities in Chile include territorial inequalities that remain unobserved when focusing exclusively on administrative regions. A grid of types of territories representing the urbanization process at a sub-regional level is proposed for the study of social inequalities from a territorial perspective. A territory is delineated by a common labor market, which is measured by commutation or territorial mobility.

Key words: inequality, urbanization, labor market, territories, commutation.

\section{Desigualdades sociais e tipos de territórios no Chile}

Resumo: As desigualdades sociais no Chile incluem as desigualdades territoriais que permanecem despercebidos quando o foco exclusivamente regiões administrativas. Um conjunto interligado de tipos representativos de territórios de urbanização em um nível sub-regional, é proposto para o estudo das desigualdades sociais a partir de uma perspectiva regional. Um território é definido por um mercado de trabalho comum, o que é medido pela comutação ou mobilidade territorial.

Palavras-chave: desigualdade, urbanização, mercado de trabalho, territórios, comutação. 


\section{Introducción}

El estudio de las desigualdades sociales desde una perspectiva territorial aporta una dimensión relevante para caracterizarlas, pero cuando se elige como nivel territorial para el análisis a las regiones administrativas, se desconoce la escala territorial donde se generan las principales diferencias, según sostendremos en este artículo. El objetivo de este trabajo es proponer un conjunto de tipos de territorios subregionales que facilite el análisis de las desigualdades sociales a nivel territorial.

Según datos de la OECD, un alto nivel de desigualdad caracteriza a Chile en comparación con otros países y las diferencias de ingresos entre las regiones del país son también superiores con respecto a la mayoría de los países integrantes de dicha organización (OECD 2009, 2011). Esto pone de relieve la importancia del estudio de las desigualdades sociales tanto a nivel nacional como sub-nacional.

El sistema administrativo chileno distingue a nivel subnacional tres tipos de unidades territoriales principales: 15 regiones, 54 provincias y 346 comunas. Los análisis sobre desigualdades sociales en el territorio chileno promovidos desde organismos públicos nacionales y organismos internacionales, han utilizado preferentemente la estructura administrativa regional (MIDEPLAN 2009, OECD 2009, CEPAL 2010). Este nivel subnacional ha sido adoptado también en estudios académicos basados en encuestas de hogares que por su representatividad estadística sólo permiten análisis detallados a nivel de regiones (Feres 2000, Contreras 2001, Pizzolito 2005, Solimano y Torche 2008).

Sin embargo, en términos espaciales las regiones jurídicamente establecidas parecen ser de una escala excesivamente amplia para reflejar la diversidad de situaciones en su interior. Más aún, las definiciones administrativas de las regiones, basadas en factores históricos, geográficos, jurídicos, políticos y de eficacia en las políticas públicas, pueden ocultar las líneas divisorias del territorio respecto de las desigualdades sociales.

Propendiendo a estudiar las desigualdades de ingresos a una escala subnacional más pequeña, se han desarrollado estudios econométricos que consideran a las comunas del país como unidad básica. Se han combinado datos censales y de encuestas de hogares para estimar desigualdades de ingresos a nivel comunal (Agostini et al 2008, Ramírez et al 2009), pero los municipios resultan ser unidades demasiado reducidas para un análisis comprehensivo.

Teniendo como referencia que las desigualdades sociales en Chile tienen un carácter multidimensional incluyendo aspectos objetivos y subjetivos (Barozet 2008), una alternativa consiste en delimitar tantos conjuntos territoriales como objetos específicos de estudio. Sin embargo, estas variadas definiciones dificultan realizar comparaciones entre una dimensión y otra, así como disponer de una base común para el análisis 
de uno u otro aspecto en particular. El estudio de las desigualdades sociales en el territorio se vería beneficiado por una clasificación de territorios a una escala intermedia entre el nivel regional y el exclusivamente comunal.

Contribuyendo a identificar una escala intermedia y enfocando el grado de ruralidad, se han combinado datos comunales sobre tamaño de la población y viajes de trabajo para distinguir territorios (Berdegué et al 2011), lo que constituye un antecedente de este trabajo.

En este artículo proponemos una representación cuantitativa de los territorios del país a un nivel subregional, basada en el estado actual del proceso de urbanización. La urbanización constituye un proceso de larga duración, que en Chile y otros países de la región se ha prolongado durante más de dos siglos. En la actualidad, Chile es un país altamente urbanizado, por encima del promedio de los países integrantes de la OECD (OECD 2009). El país se ve afectado por la urbanización de la economía en todo el mundo y el papel predominante de las grandes ciudades en la globalización, lo que acarrea como consecuencia estructural la coexistencia de territorios subnacionales prósperos con territorios de menores ventajas comparativas en la dinámica global (De Mattos 1998, 2009). Así, podríamos sostener que las desigualdades sociales vinculadas al modelo económico dominante incluyen desigualdades territoriales que son características del actual proceso de urbanización.

Con el objetivo de dar cuenta de la distribución espacial de la desigualdad, asumimos que la escala territorial donde se generan las principales diferencias corresponde al mercado laboral común a un territorio. La conformación de los mercados laborales locales puede constituir una de las mejores formas de representar el modo como la urbanización configura socialmente el territorio nacional y de qué manera sus habitantes se insertan en ese proceso. Por lo tanto, resulta crucial identificar dónde se ubican los mercados de trabajo, a un nivel subregional.

Una corriente importante de la literatura académica actual considera a los mercados de trabajo como factor distintivo de los territorios (Antikainen 2005, Fitzsimmons y Ratcliffe 2002, Coombes y Casado-Díaz 2005), como haremos en este artículo. Las metodologías utilizadas asignan una importancia preponderante al lugar donde trabajan las personas, muchas veces fuera de la comuna de residencia, para definir el área efectiva de los mercados laborales, procedimiento que aplicamos en este trabajo utilizando datos censales.

\section{Metodología y criterios de la tipología}

Con el objeto de facilitar análisis comparativos, la metodología y los criterios de delimitación de tipos de territorios, deben ser compatibles con estándares internacionales, como haremos a continuación. 
La metodología utilizada en este artículo para definir territorios considera el criterio demográfico habitualmente empleado de medir variables como la cantidad de habitantes y densidad de los lugares poblados, distinguiendo centros urbanos de diverso tamaño y otras áreas.

Adicionalmente a esas variables demográficas tradicionalmente utilizadas, el desarrollo de los sistemas de transporte ha incrementado la conectividad de las personas que residen en diversos lugares. Esta dinámica permite que los mercados de trabajo sobrepasen a menudo los lugares donde residen las personas y los límites administrativamente definidos, como los comunales. Los desplazamientos desde el lugar de residencia al lugar de trabajo, sea a la misma comuna o a otra, es decir, la movilidad territorial o conmutación por razones de trabajo, constituyen una respuesta a las necesidades de funcionamiento de una economía urbana que actúa como polo gravitacional sobre las áreas aledañas. Estos flujos pueden ser medidos y considerados un indicador del grado en que hay una interrelación económico-social entre áreas territoriales, específicamente como indicador de la presencia de un mercado de trabajo relativamente compartido en un territorio delimitado. La movilidad o conmutación por razones de trabajo ha sido medida en Chile por el Censo del año 2002, cuyos datos utilizaremos, así como por otras encuestas.

Sin embargo, este método es objeto de discusión, pues diversos estudios han destacado que la intensidad de las interacciones de personas y actividades entre diversos lugares, puede ser medida considerando otras dimensiones. Algunas mediciones procuran reflejar las formas más naturales en que las personas interactúan en el espacio, incorporando múltiples dimensiones socioeconómicas asociadas a los viajes diarios de las personas (Royuela et al 2009), modos de vida y consumo en centros comerciales (Nielsen y Hovgesen 2004) e incluso en base a las comunicaciones telefónicas (Ratti et al 2010).

En este artículo, por el contrario, asumimos que el trabajo de las personas y en consecuencia sus ingresos, son centrales para describir su integración al territorio. Además, adoptamos como nuestra la premisa de que las ciudades desempeñan un papel clave en el ordenamiento actual de los territorios en un país como Chile, ofreciendo a sus habitantes un mercado de trabajo dentro de sus límites urbanos. Con frecuencia comunas vecinas se encuentran estrechamente interconectadas a esos centros urbanos. Esta dinámica cada vez mayor, conduce a definir territorios que agrupan municipios, reconociendo que las ciudades constituyen una entidad geográfica con un significativo núcleo poblacional, a las que se vinculan comunas adyacentes que tienen un alto grado de integración con ese centro.

Un método similar se aplica en la mayor parte de los países europeos, de Estados Unidos y en general en los países que integran la OECD, para facilitar la realización de análisis socioeconómicos locales y estudios de las desigualdades territoriales (Antikainen 2005, Fitzsimmons y Ratcliffe 
2002, OECD 2002, OMB 2010). Manteniendo coherencia con esas definiciones y estándares utilizados internacionalmente, aplicamos cinco criterios para delimitar territorios:

En primer lugar, con el objeto de establecer el grado de interrelación de espacios del territorio nacional, utilizamos a las comunas como la unidad básica, tal como se ha efectuado en la mayoría de los países considerados, debido principalmente a la disponibilidad de datos empíricos para análisis comparativos.

En segundo lugar, para delimitar los territorios, es posible realizar sucesivas agregaciones de municipios o bien partir de una preselección de las comunas que constituyen los centros urbanos principales. En este trabajo partiremos de una definición establecida de los centros urbanos principales, como se efectúa en otros países (OMB 2010, Antikainen 2005). En Chile se cuenta con definiciones relativamente estandarizadas y ampliamente aplicadas especialmente con fines de planificación territorial, respecto de los límites de las grandes ciudades de Santiago, Concepción y Valparaíso, así como de las principales ciudades intermedias, desde Arica en el norte hasta Punta Arenas en el sur (SECTRA 2008). Estas ciudades intermedias cuentan con una población de al menos 50 mil habitantes y son relativamente autónomas de las grandes ciudades.

En tercer lugar, las ciudades y centros urbanos se caracterizan, además de su tamaño poblacional y densidad, por la intensidad de las interacciones de las personas y actividades al interior del área abarcada en cada caso. Un aspecto central de esa interacción consiste en formar parte de un mismo mercado de trabajo desde el punto de vista territorial, como se refleja en los viajes diarios desde el lugar de residencia al lugar de trabajo, los cuales ocurren preponderantemente al interior del área de la ciudad.

En comunas que se sitúan fuera de las grandes ciudades y de las ciudades intermedias, los viajes diarios con motivos de trabajo son también importantes, proporcionando un indicador para delimitar territorialmente los mercados laborales. En esos municipios son habituales los viajes a trabajar fuera de la comuna de residencia. La presencia de un alto grado de integración económica y social con otras comunas, de acuerdo al intercambio de empleos, puede ser medida a través de los viajes diarios con motivos de trabajo, un criterio definido internacionalmente que también aplicamos en este trabajo.

En cuarto lugar, la medida del intercambio de empleos entre dos comunas o áreas es definida como el flujo equivalente a la suma de quienes conmutan entre dos comunas, dividida por la cantidad de ocupados en la comuna más pequeña. Con el objeto de mantener una consistencia de este indicador con estándares internacionales, en nuestro análisis el nivel mínimo de intercambio de empleos que aplicamos consiste en un flujo de un 
15\%, lo que es idéntico al flujo actualmente utilizado en Estados Unidos (OMB 2010) y se sitúa en el rango del flujo entre un 15\% y un 20\% aplicado en los países europeos (Antikainen 2005). Alcanzar ese flujo mínimo implica que los ingresos de una parte relativamente importante de los trabajadores que residen en una comuna fuera del área urbana central están conectados directamente con empleos en el área central, a lo cual se agrega el efecto multiplicador e indirecto sobre las ocupaciones locales de quienes proveen servicios a estos residentes cuyos empleos se sitúan en el área urbana principal.

Finalmente, a nivel internacional se distingue la situación específica de comunas catalogadas como "rurales", al carecer de un centro habitado de un tamaño poblacional mínimo, establecido en 10.000 habitantes en el caso de Estados Unidos (Fitzsimmons y Ratcliffe 2002), límite que también aplicamos en este trabajo. Es decir, se trata de comunas relativamente pequeñas o bien su población se encuentra bastante dispersa, generalmente en pueblos, aldeas y caseríos según las definiciones del Instituto Nacional de Estadísticas (INE 2005). Algunas de estas comunas que carecen de un centro urbano, se interrelacionan con otras comunas considerando el flujo mínimo definido, mientras otras permanecen desvinculadas y las clasificamos como relativamente aisladas.

\section{Tipología de territorios}

De este modo, se hace posible formular una tipología del territorio del país partiendo de los principales centros urbanos y considerando para la distinción de territorios de las restantes comunas la densidad y el tamaño de los centros urbanos o la ausencia de éstos, así como el flujo de desplazamientos desde el lugar de residencia al lugar de trabajo.

Esto permite distinguir, en primer lugar, a las grandes ciudades de Santiago, Concepción y Valparaíso, cada una de las cuales abarca varias comunas. En segundo lugar, las ciudades intermedias que en su mayor parte consisten en una sola comuna y en algunos casos dos municipios. En tercer lugar, las restantes comunas, entre las cuales podemos distinguir considerando el flujo mínimo definido, como indicador de la presencia o ausencia de mercados de trabajo interrelacionados, a los municipios vinculados a ciudades grandes o intermedias, las comunas con centros urbanos menores que se integran entre sí y las comunas que no se conectan con otros municipios.

Entre las comunas desvinculadas de otros municipios, distinguimos las que cuentan con un centro urbano menor y las que carecen de éste pudiendo ser catalogadas como "predominantemente" rurales. Sin embargo, parte de las comunas que carecen de estos centros urbanos se vinculan a ciudades o a comunas del tipo de municipios que sí cuentan 
con centros urbanos menores. Esto es coherente con la diversidad de la ruralidad en cuanto a su grado, así como respecto del tipo de relaciones sociales entre sus habitantes, los desplazamientos a trabajar en otras comunas, los servicios disponibles y la base económica (Berdegué et al 2009 y 2011). Más aún, la presencia de población rural se encuentra en todos los tipos de territorios, incluyendo las ciudades, particularmente en sectores alejados de sus centros. Nuestra tipología enfatiza más en la urbanización que en la ruralidad atendiendo así a la creciente escala espacial de las interacciones en el mundo rural.

La principal ventaja de la tipología elaborada consiste en su capacidad de describir la forma como las comunas se relacionan con las ciudades en el sistema urbano, con relativa independencia de los límites administrativos, guardando relativa homogeneidad con métodos aplicados en otros países. El criterio principal consiste en la presencia de mercados laborales compartidos a nivel territorial, utilizando como indicador los viajes diarios con motivo de trabajo, además de criterios relacionados con el tamaño y la densidad poblacional. En el caso particular de Chile, esto permite identificar territorios subregionales, intermedios entre el nivel local y las regiones jurídica y administrativamente establecidas.

\section{Composición de los territorios}

En esta parte analizaremos la composición efectiva de los tipos de territorios definidos, de acuerdo a los datos obtenidos y nuestro análisis estadístico.

La fuente de información empleada fue la base de datos del Censo del año 2002, específicamente los datos sobre ocupación y acerca del comuna donde la persona trabaja. Partiendo de los criterios antes expuestos, el método aplicado consistió en primer lugar, en identificar las comunas integrantes de las ciudades. En segundo lugar, respecto del conjunto de las comunas del país se calcularon los flujos de conmutación, considerando a cada ciudad como un solo conjunto. Para facilitar el análisis de las comunas que se agrupan al tener flujos superiores al mínimo definido de un $15 \%$, se realizó un análisis estadístico de clusters. En tercer lugar, las comunas que no son ciudades intermedias o grandes ni se vinculan a otras comunas, fueron diferenciadas según si cuentan al menos con un centro urbano de tamaño menor en cuanto a su población.

Los resultados de acuerdo a la tipología definida se detallan en el Anexo y se aprecian sintéticamente en la tabla siguiente, donde se presentan la cantidad de territorios que se conforman, las comunas abarcadas y la cantidad de habitantes en cada tipo de territorio. 
Tabla

Tipos de territorios: cantidad de territorios, comunas y población

\begin{tabular}{lccccc}
\hline & & & & \multicolumn{3}{c}{ Población } \\
& Tipo de territorio & Territorio & Comuna & N & $\%$ \\
\hline 1 & s & 1 & 38 & 5.547 .881 & $37 \%$ \\
2 & Gran Santiago & 1 & 10 & 890.145 & $6 \%$ \\
3 & Gran Concepción & 1 & 5 & 816.179 & $5 \%$ \\
4 & Gran Valparaíso & 24 & 29 & 3.565 .073 & $24 \%$ \\
5 & Comunas vinculadas a ciudades (grandes o intermedias) & 27 & 75 & 1.170 .599 & $8 \%$ \\
6 & Comunas vinculadas a comuna con centro urbano menor & 8 & 17 & 482.553 & $3 \%$ \\
7 & Comunas desvinculadas y con centro urbano & 46 & 46 & 1.348 .521 & $9 \%$ \\
8 & Comunas desvinculadas y sin centro urbano & 126 & 126 & 1.229 .845 & $8 \%$ \\
\hline \multicolumn{2}{c}{ Total } & $\mathbf{2 3 4}$ & $\mathbf{3 4 6}$ & $\mathbf{1 5 . 0 5 0 . 7 9 6}$ & $\mathbf{1 0 0}$ \\
\hline
\end{tabular}

Fuente de datos: Censo 2002.

Los datos expuestos en la tabla muestran que las tres grandes ciudades concentran cerca de la mitad de la población del país, pero a esto se suman 24 ciudades intermedias que representan otro 24\% de los habitantes. A las ciudades grandes o intermedias se agrega un grupo numeroso de 75 comunas interrelacionadas con esas áreas urbanas, abarcando otro 8\% de la población. Más allá de los territorios vinculados a ciudades grandes o intermedias, se observa la presencia de un significativo número de comunas con centros urbanos al menos de tamaño menor que se encuentran interrelacionadas con otras comunas, abarcando a 8 conglomerados con centros urbanos menores y un 3\% de la población del país. Aparte de las comunas que conforman esos conglomerados, muchas comunas se encuentran desvinculadas de otras, distinguiéndose las 46 que cuentan con un centro urbano y las aún más numerosas que carecen de un centro urbano menor, sumando ambas un $17 \%$ de los habitantes del país.

Uno de los resultados más relevantes obtenidos, consiste en que al interior de las grandes ciudades, según nuestro análisis de los flujos de conmutación, los datos muestran que todas las comunas que las integran tienen un flujo de al menos un $15 \%$ con el conjunto de la ciudad de la que forman parte, un mínimo que en la mayor parte de los municipios de las metrópolis es superado con creces. Esto confirma la validez de considerar a estas ciudades como un solo conglomerado y medir de acuerdo a la misma pauta los flujos con las comunas que las rodean.

En otro extremo en cuanto a baja densidad poblacional, se encuentran los municipios que carecen de una entidad o localidad de al menos 10 mil habitantes. En su mayor parte corresponden a las comunas con mayor grado de aislamiento del país según criterios físicos, demográficos, de acceso a servicios públicos, económicos y político-administrativos, de acuerdo con un estudio del Instituto de Geografía de la Universidad Católica de 
Chile (SUBDERE 2008). Sin embargo, al medir el flujo de viajes al trabajo, muchas de estas comunas resultan estar integradas a los mercados de trabajo de ciudades o centros urbanos menores, sin formar parte de estas urbes pero manteniendo una relación de interdependencia.

Los resultados de la medición de flujos de conmutación considerando a las comunas como unidad básica, muestran que la ruralidad es menor que en un sentido estrictamente poblacional o estructural. De acuerdo a la definición del Instituto Nacional de Estadísticas, una localidad rural es aquella cuya población económicamente activa se emplea en un 50\% o más en actividades primarias y que no alcanza los 2.000 habitantes (INE 2005). La OECD (2009), por su parte, considera como población rural en Chile a los habitantes de localidades de menos de 5.000 habitantes. Siguiendo ambas definiciones, la población rural del país varía entre un 35\% y un 27\% respectivamente. Por otro lado, de acuerdo a la OECD (2009), considerando la ruralidad por provincias -el tercer nivel territorial según sus definiciones-, un 42\% la población habita en provincias predominantemente rurales. Desde un punto de vista relacional más que demográfico, aunque sin analizar directamente la distinción urbano-rural, nuestros resultados indican que sólo un 7\% de la población del país habita en comunas desvinculadas de otras y que carecen de un centro urbano. Pero esto no implica que las restantes comunas del país sean enteramente urbanas en un sentido poblacional o estructural, sino que sus habitantes más rurales tienen un cierto grado de integración a un territorio más amplio.

\section{Territorios e investigación sobre desigualdades sociales}

La tipología territorial elaborada y los resultados obtenidos pueden contribuir a ampliar el campo de la investigación sobre las desigualdades sociales, como examinaremos a continuación.

Desde una perspectiva metodológica, mientras mayor sea la coherencia o similitud y menor la disparidad de las áreas dentro de un territorio o tipo de territorios, mayor sería la heterogeneidad esperada entre las áreas de territorios o tipos de territorios delimitados, respecto de aspectos o variables específicas (Cörvers, Hensen, Bongaerts 2009). Aplicando esto, al controlar homogéneamente ciertas dimensiones o variables dentro de los territorios, como el sexo o la edad, sería posible comprender mejor las particularidades de las desigualdades sociales al interior de un territorio específico. Por otro lado, un análisis comparativo entre territorios puede enriquecer la comprensión de las desigualdades sociales.

Desde un punto de vista cuantitativo, se han realizado estimaciones sobre la pobreza y la desigualdad de ingresos en Chile a nivel comunal (Agostini, Brown y Góngora 2008, Modrego, Ramírez y Tartakowsky 2009, Ramírez, Tartakowsky y Modrego 2009), observándose fuertes diferencias tanto entre las comunas como al interior de los municipios. Partiendo de estos estudios, una hipótesis a verificar consiste en que la desigualdad de 
ingresos es estructural a la sociedad chilena y se reproduce en los tipos de territorio que hemos definido. Complementariamente, sin embargo, cabe preguntarse si los niveles de desigualdad de ingresos difieren entre esos diversos tipos de territorio, de modo que la desigualdad en su interior ocurre en rangos específicos a cada tipo.

Más allá de los ingresos, resultaría de interés examinar cuáles son los niveles de carencia en los diversos tipos de territorios con respecto a otras dimensiones claves que son objeto de las políticas públicas, tales como educación, salud, vivienda y empleo. También han sido estudiados los viajes diarios de una persona como una actividad asociada a las redes sociales de las que forma parte, las que en Chile influyen en las desigualdades sociales (Carrasco y Miller 2009, Carrasco y Cid-Aguayo 2012). Habitar en un tipo específico de territorio podría incidir en estas múltiples dimensiones de las desigualdades sociales.

Adicionalmente, desde una perspectiva sociológica, surgen interrogantes sobre la configuración territorial de las desigualdades en términos de una estratificación social. La identificación de mercados de trabajo territoriales y específicamente los tipos de territorios que hemos distinguido, podemos conjeturar que son definitorios de los estratos sociales presentes a nivel territorial. Esto constituye un aporte a la investigación científica sobre la estratificación social, permitiendo abordar preguntas referidas a sus particularidades a lo largo del territorio y en especial cuál es la composición de los estratos sociales en las comunas integrantes de cada tipo de territorio. A modo de ilustración, la cada vez más numerosa clase media podría tener una composición particular en la ciudad de Santiago en comparación con otras ciudades y comunas vinculadas, que también concentran parte importante de la población del país.

Contrariamente a lo anterior, las diferencias de ingresos, de bienestar social y de clases sociales, podrían ser mínimas al comparar entre los tipos de territorios que hemos distinguido. Sin embargo, esta tipología territorial facilita replantear hipótesis que sobre una base empírica permitan investigar efectos específicos del proceso de urbanización en las desigualdades sociales.

Independientemente de las diferencias cuantitativas en ámbitos específicos como los antes expuestos, cabe preguntarse si vivir en diferentes tipos de territorio enmarca los puntos de vista de las personas acerca de la existencia de desigualdades en el territorio. Por ejemplo, comparando a ese respecto la ciudad de Santiago con las otras grandes ciudades, las ciudades intermedias y las comunas vinculadas a las ciudades.

Se ha observado empíricamente la ausencia de identidades fuertes a nivel de las regiones administrativamente establecidas (Méndez 2008). Por otro lado, se ha constatado un repliegue al espacio de la vida privada de las personas (Lechner 1988), particularmente en las clases medias, sin que eso signifique una completa apatía e inactividad respecto de un interés más 
general que el individual (Méndez y Barozet 2012). Pero cabe preguntarse cómo difiere esto entre un tipo de territorio subregional y otro, incidiendo lo territorialmente particular en la forma de apreciar y establecer diferencias entre las personas.

La forma como es imaginado un territorio y construido socialmente (Dematteis y Governa 2005), podría ser mejor comprendida observando las percepciones y prácticas relativas a las diferencias sociales en su interior y frente a otros territorios.

La tipología elaborada aporta una representación cuantitativa aplicable en análisis estadísticos y proporciona un marco para estudiar las subjetividades de las personas, estratos sociales y actores en los territorios. Pero estos tipos de territorios deben entenderse como una delimitación provisional y tentativa para comprender la forma como es construido socialmente un territorio, lo que no es independiente de las desigualdades sociales en su interior y en comparación a otros territorios. 


\section{Nota}

* Este artículo ha sido posible gracias al apoyo del Proyecto Anillo Desigualdades (SOC 12 Conicyt) y del Centro de Investigación Sociedad y Políticas Públicas (CISPO) de la Universidad de Los Lagos. Los autores agradecen los aportes a este trabajo de Julio Berdegué y el equipo del Centro Latinoamericano para el Desarrollo Rural (RIMISP). 


\section{Bibliografía}

Agostini, Claudio, Philip H. Brown y Diana Paola Góngora (2008), “Distribución Espacial de la Pobreza en Chile”. Estudios de Economía, Vol. 35, N¹, junio, p. 79-110.

Antikainen, Janne (2005), “The concept of the Functional Urban Area”. Das Bundesinstitut für Bau, Stadt-und Raumforschung, Informationen zur Raumentwicklung, No. 5.

Barozet, Emmanuelle (2008), “¿De qué hablamos cuando hablamos de estratificación social en Chile? Un estado del arte.” Presentación Congreso PreAlas 2008, Universidad de Chile, Santiago, Chile.

Berdegué, Julio, Benjamín Jara, Ricardo Fuentealba, Jaime Tohá, Félix Modrego, Alexander Schejtman y Naim Bro (2011), “Territorios funcionales en Chile”. Centro Latinoamericano para el Desarrollo Rural (RIMISP), Documento de Trabajo, $N^{\circ} 102$, Santiago, Chile.

Berdegué, Julio, Esteban Jara, Félix Modrego, Ximena Sanclemente y Alexander Schejtman (2009), Ciudades Rurales en Chile. RIMISP-Centro Latinoamericano para el Desarrollo Rural, Santiago, Chile.

Carrasco, J.A. and B. Cid-Aguayo (2012), “Network capital, social networks, and travel: An empirical illustration from Concepción, Chile”. Environment and Planning A, forthcoming.

Carrasco, J.A. and E. J. Miller (2009), “The social dimension in action: A multilevel, personal networks model of social activity frequency". Transportation Research, Part A, 43(1), pp. 90-104.

CEPAL (2010), La hora de la igualdad. Brechas por cerrar, caminos por abrir. CEPAL, Santiago, Chile.

Contreras, Dante (2001), "Economic Growth and Poverty Reduction by Region: Chile 1990-96”, Development Policy Review, 19, 3.

Coombes, Mike, José M. Casado-Díaz (2005), The evolution of Local Labour Market Areas in contrasting regions. 45th Congress of the European Regional Science Association, Amsterdam.

Cörvers, Frank, Maud Hensen, Dion Bongaerts (2009), “The Delimitation and Coherence of Functional and Administrative Regions”. Regional Studies, 43, 01, p. 19-31.

De Mattos, Carlos (1998), Globalización y territorio: impactos y perspectivas. Fondo de Cultura Económica, Santiago, Chile.

Ídem (2009), “Modernización capitalista y revolución urbana en América 
Latina”. Peter Brand (comp. y ed.). La ciudad latinoamericana en el siglo XXI. Globalización, neoliberalismo, planeación. Universidad Nacional de Colombia, Medellín, pp. 41-73.

Dematteis, Giuseppe y Francesca Governa (2005), “Territorio y territorialidad en el desarrollo local. La contribución del modelo SLOT”. Boletín de la A.G.E., $\mathrm{N}^{\circ}$ 39, pp. 31-58.

Feres, Juan Carlos (2000), La pobreza en Chile en el año 2000. CEPAL, Serie Estudios Estadísticos y Prospectivos, No 14, Santiago, Chile.

Fitzsimmons, James and Michael R. Ratcliffe (2002), "Reflections on the review of the metropolitan area standards in the United States, 1990-2000". Paper prepared for the conference New Forms of Urbanization: Conceptualizing and Measuring Human Settlement in the Twenty-first Century, organized by the IUSSP Working Group on Urbanization and held at the Rockefeller Foundation's Study and Conference Center in Bellagio, Italy, March.

INE (2005), Chile: ciudades, pueblos, aldeas y caseríos. Instituto Nacional de Estadísticas, Santiago, Chile.

Lechner, Norbert (1988), Los patios interiores de la democracia. FLACSO, Santiago, Chile.

Méndez, María Luisa (2009), Proceso de descentralización e identidad regional: ¿Cómo se perciben los habitantes de regiones y cómo perciben los procesos de desarrollo regional? Proyecto Desigualdades, Documento de trabajo, Santiago, Chile.

Méndez, María Luisa, Emmanuelle Barozet (2012), “Lo auténtico también es público. Comprensión de lo público desde las clases medias en Chile.” Revista Polis, No. 31.

MIDEPLAN (2009), Distribución del ingreso. Encuesta CASEN 2009. Ministerio de Planificación, Santiago, Chile.

Modrego, Félix, Eduardo Ramírez y Andrea Tartakowsky (2009), “La heterogeneidad espacial del desarrollo económico en Chile: Radiografía a los cambios en bienestar durante la década de los 90 por estimaciones en áreas pequeñas”. Centro Latinoamericano para el Desarrollo Rural (RIMISP), Documento de Trabajo, N 9, Santiago, Chile.

Nielsen, Thomas S. \& Henrik Harder Hovgesen (2004), "Urban fields in the making: new evidence from a Danish context”. Paper for the AESOP Congress, Grenoble, France, July 1-4.

OCDE (2002), Redefining Territories: Functional Regions. OECD Publishing, París. 
Ídem (2009), Estudios territoriales de la OCDE. Chile. OECD Publishing, París.

Ídem (2011), Society at a Glance 2011: OECD Social Indicators. OECD Publishing, París.

OMB (Office of Management and Budget) (2010), "2010 Standards for Delineating Metropolitan and Micropolitan Statistical Areas”. Federal Register, Vol. 75, No. 123, June 28.

Pizzolito, G. (2005), "Monitoring Socio-Economic Conditions in Argentina, Chile, Paraguay, and Uruguay: Chile”, CEDLAS-World Bank Report, December.

Ramírez, Eduardo, Andrea Tartakowsky y Félix Modrego (2009), "La importancia de la desigualdad geográfica en Chile”. Centro Latinoamericano para el Desarrollo Rural (RIMISP), Documento de Trabajo, $N^{\circ} 30$, Santiago, Chile.

Ratti, C., S. Sobolevsky, F. Calabrese, C. Andris, J. Reades et al. (2010), "Redrawing the Map of Great Britain from a Network of Human Interactions”. PloS ONE, Volume 5, Issue 12, e14248, December.

Royuela, Vicente, Javier Romaní y Manuel Artís (2009), “Using Quality of Life Criteria to Define Urban Areas in Catalonia”. Social Indicators Research, 90, pp. 419-440.

SECTRA (2008), Actualización metodología análisis sistema de transporte de ciudades de gran tamaño y tamaño medio (MESPE). MIDEPLAN, Santiago, Chile.

Solimano, Andrés y Arístides Torche (2008), La distribución del ingreso en Chile 1987-2006: análisis y consideraciones de política. Banco Central de Chile. Documentos de Trabajo, $\mathrm{N}^{\circ}$ 480, Agosto, Santiago, Chile.

SUBDERE (Subsecretaría de Desarrollo Regional) (2008), Actualización estudio diagnóstico y propuesta para territorios aislados. Ministerio del Interior, Subsecretaría de Desarrollo Regional, Santiago, Chile.

Recibido: 05.05.2012

Aceptado: 09.01.2013 


\section{ANEXO}

Códigos de los tipos de territorio

1 Gran Santiago

2 Gran Concepción

3 Gran Valparaíso

$4 \quad$ Ciudades Intermedias

5 Comunas vinculadas a ciudades (grandes o intermedias)

6 Comunas vinculadas a comuna con centro urbano menor

7 Comunas desvinculadas y con centro urbano

8 Comunas desvinculadas y sin centro urbano

No Territorio Código Nombre Tipo de Territorio

\begin{tabular}{|c|c|c|c|}
\hline 1 & 1101 & Iquique & 4 \\
\hline 1 & 1107 & Alto Hospicio & 4 \\
\hline 2 & 1401 & Pozo Almonte & 5 \\
\hline 2 & 1405 & Pica & 5 \\
\hline 3 & 15101 & Arica & 4 \\
\hline 4 & 15102 & Camarones & 5 \\
\hline 4 & 15201 & Putre & 5 \\
\hline 4 & 15202 & General Lagos & 5 \\
\hline 5 & 1402 & Camiña & 8 \\
\hline 6 & 1404 & Huara & 8 \\
\hline 7 & 1403 & Colchane & 8 \\
\hline 8 & 2101 & Antofagasta & 4 \\
\hline 9 & 2102 & Mejillones & 5 \\
\hline 9 & 2103 & Sierra Gorda & 5 \\
\hline 9 & 2104 & Taltal & 5 \\
\hline 10 & 2201 & Calama & 4 \\
\hline 11 & 2202 & Ollagüe & 5 \\
\hline 12 & 2203 & San Pedro de Atacama & 8 \\
\hline 13 & 2301 & Tocopilla & 7 \\
\hline 14 & 2302 & María Elena & 8 \\
\hline 15 & 3101 & Copiapó & 4 \\
\hline 16 & 3103 & Tierra Amarilla & 8 \\
\hline 17 & 3301 & Vallenar & 6 \\
\hline 17 & 3304 & Huasco & 6 \\
\hline
\end{tabular}




\begin{tabular}{|c|c|c|c|}
\hline 18 & 3102 & Caldera & 7 \\
\hline 19 & 3201 & Chañaral & 7 \\
\hline 20 & 3302 & Alto del Carmen & 8 \\
\hline 21 & 3303 & Freirina & 8 \\
\hline 22 & 3202 & Diego de Almagro & 8 \\
\hline 23 & 4101 & La Serena & 4 \\
\hline 23 & 4102 & Coquimbo & 4 \\
\hline 24 & 4103 & Andacollo & 5 \\
\hline 24 & 4104 & La Higuera & 5 \\
\hline 24 & 4106 & Vicuña & 5 \\
\hline 25 & 4301 & Ovalle & 4 \\
\hline 26 & 4304 & Punitaqui & 5 \\
\hline 27 & 4105 & Paiguano & 8 \\
\hline 28 & 4201 & Illapel & 7 \\
\hline 29 & 4204 & Salamanca & 7 \\
\hline 30 & 4202 & Canela & 8 \\
\hline 31 & 4203 & Los Vilos & 7 \\
\hline 32 & 4303 & Monte Patria & 8 \\
\hline 33 & 4305 & Río Hurtado & 8 \\
\hline 34 & 4302 & Combarbalá & 8 \\
\hline 35 & 5101 & Valparaíso & 3 \\
\hline 35 & 5103 & Concón & 3 \\
\hline 35 & 5106 & Quilpué & 3 \\
\hline 35 & 5108 & Villa Alemana & 3 \\
\hline 35 & 5109 & Viña del Mar & 3 \\
\hline 36 & 5102 & Casablanca & 5 \\
\hline 36 & 5105 & Puchuncaví & 5 \\
\hline 36 & 5107 & Quintero & 5 \\
\hline 37 & 5104 & Juan Fernández & 8 \\
\hline 38 & 5201 & Isla de Pascua & 8 \\
\hline 39 & 5301 & Los Andes & 4 \\
\hline 40 & 5302 & Calle Larga & 5 \\
\hline 40 & 5303 & Rinconada & 5 \\
\hline 40 & 5304 & San Esteban & 5 \\
\hline 41 & 5401 & La Ligua & 6 \\
\hline 41 & 5403 & Papudo & 6 \\
\hline 42 & 5404 & Petorca & 8 \\
\hline 43 & 5501 & Quillota & 6 \\
\hline 43 & 5504 & La Cruz & 6 \\
\hline 44 & 5502 & Calera & 6 \\
\hline 44 & 5503 & Hijuelas & 6 \\
\hline
\end{tabular}


Polis, Revista Latinoamericana, Volumen 12, No 34, 2013

\begin{tabular}{|c|c|c|c|}
\hline 44 & 5506 & Nogales & 6 \\
\hline 45 & 5505 & Limache & 6 \\
\hline 45 & 5507 & Olmué & 6 \\
\hline 46 & 5601 & San Antonio & 4 \\
\hline 47 & 5603 & Cartagena & 5 \\
\hline 47 & 5606 & Santo Domingo & 5 \\
\hline 48 & 5701 & San Felipe & 4 \\
\hline 49 & 5704 & Panquehue & 5 \\
\hline 49 & 5705 & Putaendo & 5 \\
\hline 49 & 5706 & Santa María & 5 \\
\hline 50 & 5602 & Algarrobo & 8 \\
\hline 51 & 5604 & El Quisco & 8 \\
\hline 52 & 5702 & Catemu & 8 \\
\hline 53 & 5703 & Llaillay & 7 \\
\hline 54 & 5402 & Cabildo & 7 \\
\hline 55 & 5405 & Zapallar & 8 \\
\hline 56 & 5605 & El Tabo & 8 \\
\hline 57 & 6101 & Rancagua & 4 \\
\hline 57 & 6108 & Machalí & 4 \\
\hline 58 & 6102 & Codegua & 5 \\
\hline 58 & 6103 & Coinco & 5 \\
\hline 58 & 6105 & Doñihue & 5 \\
\hline 58 & 6106 & Graneros & 5 \\
\hline 58 & 6111 & Olivar & 5 \\
\hline 58 & 6116 & Requínoa & 5 \\
\hline 59 & 6109 & Malloa & 6 \\
\hline 59 & 6115 & Rengo & 6 \\
\hline 60 & 6301 & San Fernando & 6 \\
\hline 60 & 6308 & Placilla & 6 \\
\hline 61 & 6306 & Palmilla & 8 \\
\hline 62 & 6310 & Santa Cruz & 7 \\
\hline 63 & 6104 & Coltauco & 8 \\
\hline 64 & 6107 & Las Cabras & 8 \\
\hline 65 & 6112 & Peumo & 8 \\
\hline 66 & 6204 & Marchihue & 8 \\
\hline 67 & 6307 & Peralillo & 8 \\
\hline 68 & 6309 & Pumanque & 8 \\
\hline 69 & 6110 & Mostazal & 7 \\
\hline 70 & 6117 & San Vicente & 7 \\
\hline 71 & 6114 & Quinta de Tilcoco & 8 \\
\hline 72 & 6113 & Pichidegua & 8 \\
\hline
\end{tabular}




\begin{tabular}{|c|c|c|c|}
\hline 73 & 6201 & Pichilemu & 8 \\
\hline 74 & 6202 & La Estrella & 8 \\
\hline 75 & 6203 & Litueche & 8 \\
\hline 76 & 6205 & Navidad & 8 \\
\hline 77 & 6206 & Paredones & 8 \\
\hline 78 & 6303 & Chimbarongo & 7 \\
\hline 79 & 6305 & Nancagua & 8 \\
\hline 80 & 6302 & Chépica & 8 \\
\hline 81 & 6304 & Lolol & 8 \\
\hline 82 & 7101 & Talca & 4 \\
\hline 83 & 7105 & Maule & 5 \\
\hline 83 & 7106 & Pelarco & 5 \\
\hline 83 & 7107 & Pencahue & 5 \\
\hline 83 & 7109 & San Clemente & 5 \\
\hline 83 & 7110 & San Rafael & 5 \\
\hline 84 & 7301 & Curicó & 4 \\
\hline 85 & 7304 & Molina & 5 \\
\hline 85 & 7305 & Rauco & 5 \\
\hline 85 & 7306 & Romeral & 5 \\
\hline 85 & 7308 & Teno & 5 \\
\hline 86 & 7102 & Constitución & 7 \\
\hline 87 & 7104 & Empedrado & 7 \\
\hline 88 & 7202 & Chanco & 7 \\
\hline 89 & 7103 & Curepto & 8 \\
\hline 90 & 7108 & Río Claro & 8 \\
\hline 91 & 7201 & Cauquenes & 7 \\
\hline 92 & 7203 & Pelluhue & 8 \\
\hline 93 & 7307 & Sagrada Familia & 8 \\
\hline 94 & 7302 & Hualañé & 8 \\
\hline 95 & 7303 & Licantén & 8 \\
\hline 96 & 7309 & Vichuquén & 8 \\
\hline 97 & 7401 & Linares & 7 \\
\hline 98 & 7402 & Colbún & 8 \\
\hline 99 & 7403 & Longaví & 8 \\
\hline 100 & 7408 & Yerbas Buenas & 8 \\
\hline 101 & 7404 & Parral & 7 \\
\hline 102 & 7405 & Retiro & 8 \\
\hline 103 & 7406 & San Javier & 7 \\
\hline 104 & 7407 & Villa Alegre & 8 \\
\hline 105 & 8101 & Concepción & 2 \\
\hline 105 & 8102 & Coronel & 2 \\
\hline
\end{tabular}


Polis, Revista Latinoamericana, Volumen 12, No 34, 2013

\begin{tabular}{|c|c|c|c|}
\hline 105 & 8103 & Chiguayante & 2 \\
\hline 105 & 8105 & Hualqui & 2 \\
\hline 105 & 8106 & Lota & 2 \\
\hline 105 & 8107 & Penco & 2 \\
\hline 105 & 8108 & San Pedro de la Paz & 2 \\
\hline 105 & 8110 & Talcahuano & 2 \\
\hline 105 & 8111 & Tomé & 2 \\
\hline 105 & 8112 & Hualpén & 2 \\
\hline 106 & 8104 & Florida & 5 \\
\hline 106 & 8109 & Santa Juana & 5 \\
\hline 106 & 8202 & Arauco & 5 \\
\hline 106 & 8308 & Quilaco & 5 \\
\hline 107 & 8301 & Los Ángeles & 4 \\
\hline 108 & 8302 & Antuco & 5 \\
\hline 108 & 8307 & Negrete & 5 \\
\hline 108 & 8309 & Quilleco & 5 \\
\hline 108 & 8311 & Santa Bárbara & 5 \\
\hline 109 & 8304 & Laja & 6 \\
\hline 109 & 8310 & San Rosendo & 6 \\
\hline 110 & 8401 & Chillán & 4 \\
\hline 110 & 8406 & Chillán Viejo & 4 \\
\hline 111 & 8405 & Coihueco & 5 \\
\hline 111 & 8411 & Pinto & 5 \\
\hline 111 & 8419 & San Nicolás & 5 \\
\hline 112 & 8306 & Nacimiento & 7 \\
\hline 113 & 8201 & Lebu & 7 \\
\hline 114 & 8205 & Curanilahue & 7 \\
\hline 115 & 8203 & Cañete & 7 \\
\hline 116 & 8207 & Tirúa & 8 \\
\hline 117 & 8204 & Contulmo & 8 \\
\hline 118 & 8206 & Los Alamos & 7 \\
\hline 119 & 8305 & Mulchén & 7 \\
\hline 120 & 8303 & Cabrero & 7 \\
\hline 121 & 8312 & Tucapel & 8 \\
\hline 122 & 8421 & Yungay & 8 \\
\hline 123 & 8313 & Yumbel & 8 \\
\hline 124 & 8314 & Alto BiobÍo & 8 \\
\hline 125 & 8402 & Bulnes & 7 \\
\hline 126 & 8407 & El Carmen & 8 \\
\hline 127 & 8408 & Ninhue & 8 \\
\hline 128 & 8412 & Portezuelo & 8 \\
\hline
\end{tabular}




\begin{tabular}{|c|c|c|c|}
\hline 129 & 8416 & San Carlos & 7 \\
\hline 130 & 8418 & San Ignacio & 8 \\
\hline 131 & 8404 & Coelemu & 8 \\
\hline 132 & 8420 & Treguaco & 8 \\
\hline 133 & 8403 & Cobquecura & 8 \\
\hline 134 & 8409 & Ñiquén & 8 \\
\hline 135 & 8410 & Pemuco & 8 \\
\hline 136 & 8413 & Quillón & 8 \\
\hline 137 & 8414 & Quirihue & 8 \\
\hline 138 & 8415 & Ránquil & 8 \\
\hline 139 & 8417 & San Fabián & 8 \\
\hline 140 & 9101 & Temuco & 4 \\
\hline 140 & 9112 & Padre las Casas & 4 \\
\hline 141 & 9105 & Freire & 5 \\
\hline 141 & 9108 & Lautaro & 5 \\
\hline 141 & 9119 & Vilcún & 5 \\
\hline 142 & 9111 & Nueva Imperial & 7 \\
\hline 143 & 9114 & Pitrufquén & 7 \\
\hline 144 & 9102 & Carahue & 8 \\
\hline 145 & 9103 & Cunco & 8 \\
\hline 146 & 9107 & Gorbea & 8 \\
\hline 147 & 9113 & Perquenco & 8 \\
\hline 148 & 9104 & Curarrehue & 8 \\
\hline 149 & 9115 & Pucón & 7 \\
\hline 150 & 9120 & Villarrica & 7 \\
\hline 151 & 9106 & Galvarino & 8 \\
\hline 152 & 9109 & Loncoche & 7 \\
\hline 153 & 9110 & Melipeuco & 8 \\
\hline 154 & 9116 & Saavedra & 8 \\
\hline 155 & 9117 & Teodoro Schmidt & 8 \\
\hline 156 & 9118 & Toltén & 8 \\
\hline 157 & 9121 & Cholchol & 8 \\
\hline 158 & 9201 & Angol & 4 \\
\hline 159 & 9202 & Collipulli & 7 \\
\hline 160 & 9206 & Los Sauces & 8 \\
\hline 161 & 9209 & Renaico & 8 \\
\hline 162 & 9203 & Curacautín & 7 \\
\hline 163 & 9204 & Ercilla & 8 \\
\hline 164 & 9205 & Lonquimay & 8 \\
\hline 165 & 9207 & Lumaco & 8 \\
\hline 166 & 9208 & Purén & 8 \\
\hline
\end{tabular}


Polis, Revista Latinoamericana, Volumen 12, No 34, 2013

\begin{tabular}{|c|c|c|c|}
\hline 167 & 9210 & Traiguén & 7 \\
\hline 168 & 9211 & Victoria & 7 \\
\hline 169 & 14101 & Valdivia & 4 \\
\hline 170 & 14105 & Máfil & 5 \\
\hline 171 & 14102 & Corral & 8 \\
\hline 172 & 14106 & Mariquina & 8 \\
\hline 173 & 14107 & Paillaco & 8 \\
\hline 174 & 14103 & Lanco & 8 \\
\hline 175 & 14104 & Los Lagos & 8 \\
\hline 176 & 14108 & Panguipulli & 7 \\
\hline 177 & 14201 & La Unión & 7 \\
\hline 178 & 14202 & Futrono & 8 \\
\hline 179 & 14203 & Lago Ranco & 8 \\
\hline 180 & 14204 & Río Bueno & 7 \\
\hline 181 & 10101 & Puerto Montt & 4 \\
\hline 182 & 10109 & Puerto Varas & 5 \\
\hline 183 & 10201 & Castro & 4 \\
\hline 184 & 10203 & Chonchi & 5 \\
\hline 184 & 10205 & Dalcahue & 5 \\
\hline 185 & 10301 & Osorno & 4 \\
\hline 186 & 10307 & San Pablo & 8 \\
\hline 187 & 10107 & Llanquihue & 7 \\
\hline 188 & 10204 & Curaco de Vélez & 8 \\
\hline 189 & 10304 & Puyehue & 8 \\
\hline 190 & 10102 & Calbuco & 7 \\
\hline 191 & 10103 & Cochamó & 8 \\
\hline 192 & 10104 & Fresia & 8 \\
\hline 193 & 10105 & Frutillar & 8 \\
\hline 194 & 10106 & Los Muermos & 8 \\
\hline 195 & 10108 & Maullín & 8 \\
\hline 196 & 10202 & Ancud & 4 \\
\hline 197 & 10206 & Puqueldón & 8 \\
\hline 198 & 10207 & Queilén & 8 \\
\hline 199 & 10208 & Quellón & 7 \\
\hline 200 & 10209 & Quemchi & 8 \\
\hline 201 & 10210 & Quinchao & 8 \\
\hline 202 & 10302 & Puerto Octay & 8 \\
\hline 203 & 10303 & Purranque & 7 \\
\hline 204 & 10305 & Río Negro & 8 \\
\hline 205 & 10306 & San Juan de La Costa & 8 \\
\hline 206 & 10401 & Chaitén & 8 \\
\hline
\end{tabular}




\begin{tabular}{|c|c|c|c|}
\hline 207 & 10402 & Futaleufú & 8 \\
\hline 208 & 10403 & Hualaihué & 8 \\
\hline 209 & 10404 & Palena & 8 \\
\hline 210 & 11101 & Coihaique & 4 \\
\hline 211 & 11102 & Lago Verde & 5 \\
\hline 212 & 11202 & Cisnes & 8 \\
\hline 213 & 11203 & Guaitecas & 8 \\
\hline 214 & 11301 & Cochrane & 8 \\
\hline 215 & 11303 & Tortel & 8 \\
\hline 216 & 11402 & Río Ibáñez & 8 \\
\hline 217 & 11201 & Aisén & 7 \\
\hline 218 & 11401 & Chile Chico & 8 \\
\hline 219 & 11302 & O’Higgins & 8 \\
\hline 220 & 12101 & Punta Arenas & 4 \\
\hline 221 & 12102 & Laguna Blanca & 5 \\
\hline 221 & 12103 & Río Verde & 5 \\
\hline 221 & 12104 & San Gregorio & 5 \\
\hline 221 & 12202 & Antártica & 5 \\
\hline 221 & 12302 & Primavera & 5 \\
\hline 221 & 12401 & Natales & 5 \\
\hline 221 & 12402 & Torres del Paine & 5 \\
\hline 222 & 12201 & Cabo de Hornos & 8 \\
\hline 223 & 12301 & Porvenir & 8 \\
\hline 224 & 12303 & Timaukel & 8 \\
\hline 225 & 13101 & Santiago & 1 \\
\hline 225 & 13102 & Cerrillos & 1 \\
\hline 225 & 13103 & Cerro Navia & 1 \\
\hline 225 & 13104 & Conchalí & 1 \\
\hline 225 & 13105 & El Bosque & 1 \\
\hline 225 & 13106 & Estación Central & 1 \\
\hline 225 & 13107 & Huechuraba & 1 \\
\hline 225 & 13108 & Independencia & 1 \\
\hline 225 & 13109 & La Cisterna & 1 \\
\hline 225 & 13110 & La Florida & 1 \\
\hline 225 & 13111 & La Granja & 1 \\
\hline 225 & 13112 & La Pintana & 1 \\
\hline 225 & 13113 & La Reina & 1 \\
\hline 225 & 13114 & Las Condes & 1 \\
\hline 225 & 13115 & Lo Barnechea & 1 \\
\hline 225 & 13116 & Lo Espejo & 1 \\
\hline 225 & 13117 & Lo Prado & 1 \\
\hline \multicolumn{4}{|c|}{489} \\
\hline
\end{tabular}


Polis, Revista Latinoamericana, Volumen 12, No 34, 2013

\begin{tabular}{|c|c|c|c|}
\hline 225 & 13118 & Macul & 1 \\
\hline 225 & 13119 & Maipú & 1 \\
\hline 225 & 13120 & Ñuñoa & 1 \\
\hline 225 & 13121 & Pedro Aguirre Cerda & 1 \\
\hline 225 & 13122 & Peñalolén & 1 \\
\hline 225 & 13123 & Providencia & 1 \\
\hline 225 & 13124 & Pudahuel & 1 \\
\hline 225 & 13125 & Quilicura & 1 \\
\hline 225 & 13126 & Quinta Normal & 1 \\
\hline 225 & 13127 & Recoleta & 1 \\
\hline 225 & 13128 & Renca & 1 \\
\hline 225 & 13129 & San Joaquín & 1 \\
\hline 225 & 13130 & San Miguel & 1 \\
\hline 225 & 13131 & San Ramón & 1 \\
\hline 225 & 13132 & Vitacura & 1 \\
\hline 225 & 13201 & Puente Alto & 1 \\
\hline 225 & 13202 & Pirque & 1 \\
\hline 225 & 13301 & Colina & 1 \\
\hline 225 & 13302 & Lampa & 1 \\
\hline 225 & 13401 & San Bernardo & 1 \\
\hline 225 & 13403 & Calera de Tango & 1 \\
\hline 226 & 13402 & Buin & 5 \\
\hline 226 & 13404 & Paine & 5 \\
\hline 227 & 13601 & Talagante & 5 \\
\hline 227 & 13602 & El Monte & 5 \\
\hline 227 & 13603 & Isla de Maipo & 5 \\
\hline 227 & 13604 & Padre Hurtado & 5 \\
\hline 227 & 13605 & Peñaflor & 5 \\
\hline 228 & 13203 & San José de Maipo & 5 \\
\hline 229 & 13303 & Tiltil & 5 \\
\hline 230 & 13501 & Melipilla & 7 \\
\hline 231 & 13504 & María Pinto & 8 \\
\hline 232 & 13505 & San Pedro & 8 \\
\hline 233 & 13502 & Alhué & 8 \\
\hline 234 & 13503 & Curacaví & 5 \\
\hline
\end{tabular}

